\title{
A molecular dynamics investigation of the deformation mechanism and shape memory effect of epoxy shape memory polymers ${ }^{\dagger}$
}

\author{
Hua Yang ${ }^{1}$, ZhengDao Wang ${ }^{1}$, YaFang Guo ${ }^{1 *}$, and XingHua Shi ${ }^{2 *}$ \\ ${ }^{1}$ Institute of Engineering Mechanics, Beijing Jiaotong University, Beijing 100044, China; \\ ${ }^{2}$ State Key Laboratory of Nonlinear Mechanics, Institute of Mechanics, Chinese Academy of Sciences, Beijing 100190, China
}

Received August 25, 2015; accepted November 2, 2015; published online January 6, 2016

\begin{abstract}
Following deformation, thermally induced shape memory polymers (SMPs) have the ability to recover their original shape with a change in temperature. In this work, the thermomechanical properties and shape memory behaviors of three types of epoxy SMPs with varying curing agent contents were investigated using a molecular dynamics (MD) method. The mechanical properties under uniaxial tension at different temperatures were obtained, and the simulation results compared reasonably with experimental data. In addition, in a thermomechanical cycle, ideal shape memory effects for the three types of SMPs were revealed through the shape frozen and shape recovery responses at low and high temperatures, respectively, indicating that the recovery time is strongly influenced by the ratio of E-51 to 4,4'-Methylenedianiline.
\end{abstract}

shape memory polymer, molecular dynamics simulation, thermomechanics

PACS number(s): 61.41.+e, 02.70.Ns, 62.20.-x

Citation: H. Yang, Z. D. Wang, Y. F. Guo, and X. H. Shi, A molecular dynamics investigation of the deformation mechanism and shape memory effect of epoxy shape memory polymers, Sci. China-Phys. Mech. Astron. 59, 634601 (2016), doi: 10.1007/s11433-015-5758-4

\section{Introduction}

Shape memory polymers (SMPs), which are an important type of stimuli-responsive polymer, can recover their original shape upon exposure to external stimuli, such as heat [1], light [2-4], magnetic field [5,6], and moisture [7,8]. Owing to their prominent advantages, e.g., low density, high frozen strain, low manufacturing cost, easy processability, wide shape transition temperature, and partial biocompatibility, SMPs have wide potential applications, such as in actuators, sensors, and smart devices. During the last two decades, extensive attention has been paid to the fabrication and design of new SMPs and their composites, including SMP matrices [9-13], particle- or fiber-reinforced SMP compo-

*Corresponding authors (YaFang Guo, email: yfguo@bjtu.edu.cn;

XingHua Shi, email: shixh@imech.ac.cn)

$\uparrow$ Recommended by YouShi Hong (Associate Editor-in-Chief) sites [13,14], SMP foams $[15,16]$, and sandwich structures $[17,18]$. Meanwhile, numerous works have reported the thermomechanical behavior and shape memory effects of SMPs and their composites [19-22]. Based on experimental results and the molecular mechanism of shape memory, $\mathrm{Lu}$ et al. $[23,24]$ presented a phenomenological approach to study the state transition and working mechanism of the chemoresponsive shape memory effect in SMPs. In addition, Liu et al. [24] developed a three-dimensional small-strain internal state variable constitutive model to predict the thermomechanical behavior of SMPs. Recently, SMPs with two-way and multishape memory effects are attracting ongoing interest [25-28]. In these studies, the ratio of curing agent to epoxy resin is thought to be an essential factor in the fabrication of high-performance SMPs because different ratios of curing agent to epoxy resin lead to different glass transition temperatures, different thermomechanical properties, etc. However, an understanding of how the ratio affects 
these properties remains elusive. Therefore, we built three SMP epoxy systems with varying curing agent contents to investigate the influence of the ratio of curing agent to epoxy resin on the thermomechanical properties of SMP, as well as their recovery properties.

Molecular dynamics (MD) is now a well-established and important tool that allows practical problems to be explored in detail on the macromolecular scale for polymers to obtain information that cannot be extracted directly from experiments. MD simulations can also consider the vibrational modes of individual atoms and calculate many fundamental atomic interactions at the individual chain level, which cannot be obtained by other numerical simulation methods, such as the finite element method. For example, Jang et al. [29] used MD simulations to investigate the mechanical and transport properties of a poly(ethylene oxide)-poly(acrylic acid) double network hydrogel with $76 \mathrm{wt} \%$ water content. Li et al. [30] extensively characterized the thermomechanical response of a thermoset polymer composed of epoxy EPON862 and curing agent Diethyltoluenediamine (DETDA) using MD simulations. However, few of these simulated works involve SMPs. Diani and Gall [31] obtained some interesting results by using a full atomistic simulation of the shape-memory behavior of polyisoprene. Instead of real SMPs, a virtual polyisoprene was constructed in their model, in which entropy dependent shape storage and recovery was confirmed. Recently, Ghobadi et al. [32] presented an atomistic modeling approach to predict the shape-memory behavior of poly(L-lactide) and its cyclic uniaxial thermomechanical test.

SMP-resin-based composites reinforced by continuous fibers are regarded as the most promising materials for deployable structures in future space applications [33-36]. The fabrication of high-performance SMP epoxy systems is thus essential. A series of shape memory epoxy systems with tailored shape transition temperatures and different thermomechanical properties have been synthesized. Many experimental measurements, such as thermal frozen/recovery tests, differential scanning calorimetry (DSC), and dynamic mechanical analysis (DMA), have been performed to investigate the shape memory behavior and thermomechanical properties of the fabricated specimens. In the present work,
MD simulation was applied to study the thermomechanical properties and shape memory effect of epoxy SMPs with varying contents of curing agent. The simulation results are also compared with related experimental data [37]. Our investigation allows elucidation of the underlying mechanism that accounts for such thermomechanical properties and shape memory effect.

\section{Materials and simulation description}

The mechanical properties and shape memory behavior of three types of epoxy SMPs with varying curing agent contents were investigated in this work. The specimens were prepared using a commercial thermoset epoxy system, E-51, and curing agent, 4,4'-methylenedianiline (DDM). According to the different mass ratios of E-51 to DDM, three types of specimens were prepared. Table 1 lists the formulations of the prepared shape memory epoxy systems, labeled 1\# to 3\#. The experimental shape frozen/recovery responses and viscoelastic behaviors of specimens $1 \#$ to $3 \#$ can be found in ref. [37].

Materials Studio [38] was used to build the initial molecular model, and further MD simulation was performed using LAMMPS [39]. The E-51 and DDM repeat units are shown in Figure 1, where the letters $h$ and $t$ represent a head and a tail linkage, respectively. According to the mass ratios listed in Table 1, the repeat unit ratios of E-51 and DDM for specimens $1 \#$ to $3 \#$ are 10:3, 3:1, and 13:5, respectively. To reduce the molecular weight effect in the three systems, the molecular numbers of E-51:DDM in each system were 70:21, 66:22, and 65:25. Using the random copolymer builder and amorphous cell generator program in Materials Studio, an E-51/DDM random copolymer system was constructed.

The following dynamics simulations were all performed using LAMMPS parallel MD code. 3-D periodic boundary conditions were employed for all the simulations to remove possible surface effects. The initial equilibrated structure was obtained using NVT and NPT ensembles sequentially at a high temperature $(550 \mathrm{~K})$ with a timestep of $1 \mathrm{fs}$. Simulations were performed using polymer consistent force field

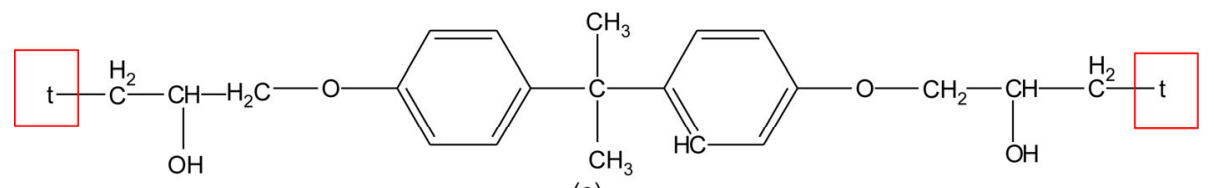

(a)

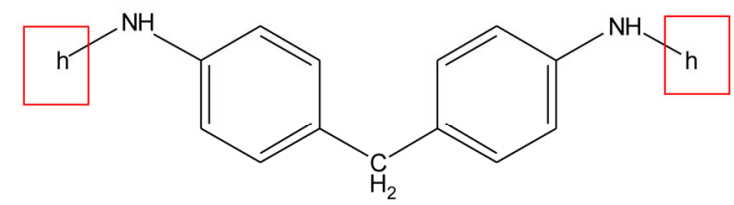

(b)

Figure 1 Molecular structural repeat units for (a) E-51 epoxy and (b) DDM curing agent. 
Table 1 Formulations of the prepared shape memory epoxy systems (mass ratio)

\begin{tabular}{ccc}
\hline Specimen & E-51 & DDM \\
\hline $1 \#$ & 100 & 15 \\
$2 \#$ & 100 & 17 \\
$3 \#$ & 100 & 19 \\
\hline
\end{tabular}

(PCFF), which is parameterized for a large class of organic molecules involving $\mathrm{H}, \mathrm{C}, \mathrm{O}, \mathrm{S}, \mathrm{P}, \mathrm{F}, \mathrm{Cl}$, and $\mathrm{Br}$, allowing it to be applied to synthetic polymers [40]. It has been found that the PCFF leads to accurate geometries for various polymeric systems. The cutoff distance was set to $9.5 \AA$ $[31,41]$. The shape memory effect in SMPs arises from the glass transition of the materials owing to the change of material temperature. All simulations in this work focused on the glassy (below the glass transition temperature) and rubbery (above the glass transition temperature) states of the materials.

\section{Results and discussion}

\subsection{Glass transition temperature}

The shape fixity and recovery responses of SMPs are due to frozen segment chains at lower temperatures and a recovery of flexibility at elevated temperatures. Such processes are determined by the glass transition temperatures $\left(T_{\mathrm{g}}\right)$ of the materials. Thus, $T_{\mathrm{g}}$ is one of the most important parameters for SMPs. The prediction of $T_{\mathrm{g}}$ by MD simulation provides an insight into the molecular structures during the shape frozen/recovery response of SMPs.

To determine $T_{\mathrm{g}}$ for the three types of specimens with different mass ratios, relaxed specimens $1 \#$ to $3 \#$ were first obtained at $550 \mathrm{~K}$. Then, an NPT ensemble was used to generate $V-T$ curves at a fixed pressure. The volumes at each temperature were obtained by cooling the system with a decrement of $50 \mathrm{~K}$. Figure 2 shows the MD simulation results for the dependence of the volume of specimens $1 \#$ to $3 \#$ on temperature. The $T_{\mathrm{g}}$ values of the materials can be determined from the intersection of the two straight lines, as shown in Figure 3 . The $T_{\mathrm{g}}$ values obtained from the MD simulations are 351,365 , and $403 \mathrm{~K}$ for specimens $1 \#, 2 \#$, and $3 \#$, respectively. Experimental values determined by differential scanning calorimetry (DSC) $(329,353$, and 379 $\mathrm{K}$ for specimens 1\#, 2\#, and 3\#, respectively [37]) are also presented in Figure 3 (red dotted lines). The results show that the simulated results are close to the experimental data, with higher curing agent content leading to higher $T_{\mathrm{g}}$ values. The slight difference between the numerical and experimental results may be due to the much smaller chain number and considerably higher cooling rate in the MD simulations.

\subsection{Uniaxial deformation}

It is possible to obtain the uniaxial tension stress-strain curves for amorphous polymers below and above the glass transition. Here, MD simulations of uniaxial tensile tests were carried out on specimens $1 \#$ to $3 \#$ at different temperatures. The uniaxial tensile deformation is carried out by continuously increasing the length of the simulation box along $x$ direction. A Berendsen barostat was used to maintain a pressure of zero in the direction perpendicular to the stretching direction. The stress-strain curves of $y$ - and $z$-directions of 1\# sample are shown in Figure 3(a), which indicating that the stress remains to be $0 \mathrm{MPa}$ during the tension. The stress-strain curves of the stretched direction are shown in Figure 3(b). In present study, the samples were stretched to $50 \%$ strain with a loading strain rate of $5 \times 10^{9}$ $\mathrm{s}^{-1}$. The results in Figure 3 show that changes in temperature have a very clear effect on the stress-strain response. As expected, the material stiffness decreases with increasing temperature. Following the full chain model of $\mathrm{Wu}$ and van der Giessen $[42,43]$ and assuming that chains are randomly distributed, the average chain orientation under a uniaxial stretching can be calculated as:

$$
\bar{\theta}==\int_{0}^{\pi / 2} \tan ^{-1}\left(\frac{1}{\lambda_{1}^{3 / 2}} \tan \theta\right) \sin \theta \mathrm{d} \theta,
$$

where $\lambda_{1}$ is the stretch and the elastomer is assumed to be incompressible. The average chain stretch can be calculated as:
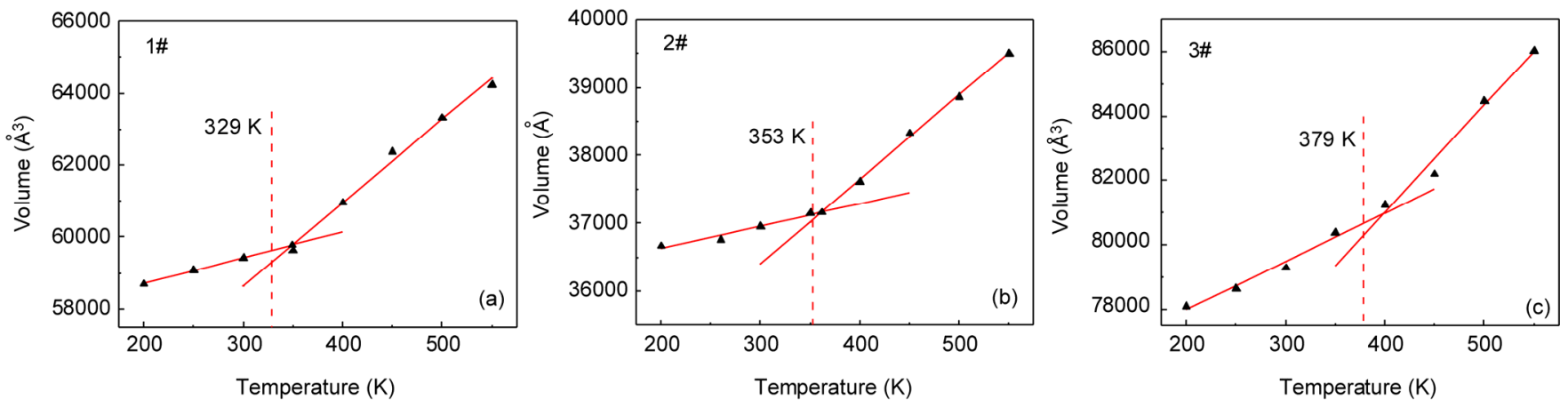

Figure 2 (Color online) Volume of specimens 1\# (a), 2\# (b), and 3\# (c) as a function of temperature. Experimental data taken from ref. [37] are indicated with red dotted lines. 


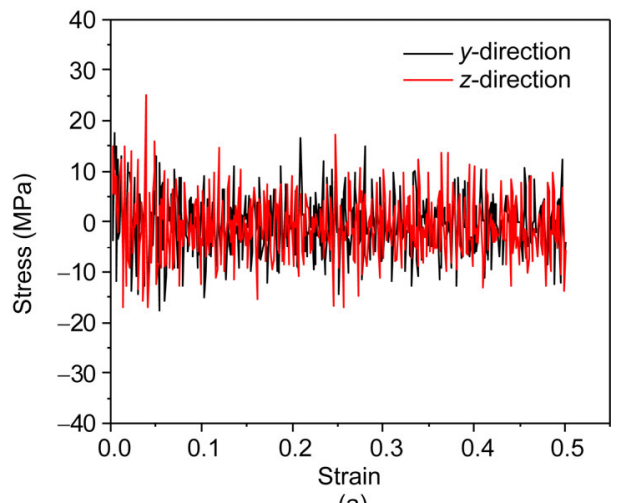

(a)
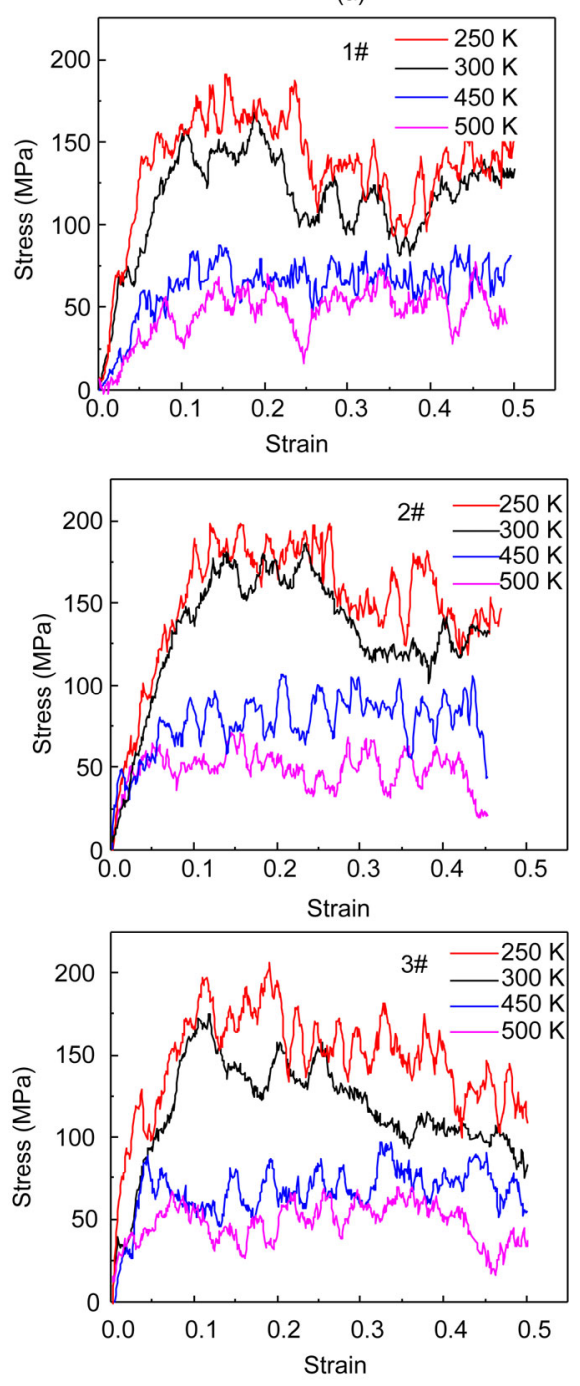

(b)

Figure 3 (Color online) (a) Stress-strain curves of $y$ - and $z$-directions of 1\# sample; (b) stress-strain curves of specimens 1\#, 2\# and 3\# at different temperatures.

$$
\bar{\lambda}==\int_{0}^{\pi / 2} \sqrt{\left(\lambda_{1} \cos \theta\right)^{2}+\lambda_{1}^{-1} \sin ^{2} \theta} \sin \theta \mathrm{d} \theta
$$

The chain angle in the loading direction and end-to-end distance under uniaxial tension were calculated to represent the chain orientation and chain stretch, respectively. Figure 4 shows a comparison between the theoretical prediction and the MD simulations, and the MD simulation can be seen to follow theoretical predictions well.

The simulated stress-strain behavior at a strain rate of $5 \times 10^{9} \mathrm{~s}^{-1}$ shows the same trends as those observed in experimental testing for amorphous polymers. At 250 and $300 \mathrm{~K}$, the stress-strain curves show a typical elastic response followed by yielding, softening, and hardening. The yield point and softening regime is much more pronounced at 250 $\mathrm{K}$. At $400 \mathrm{~K}$ (above $T_{\mathrm{g}}$ ), the stress-strain curve does not show any softening or subsequent strong hardening. The total interaction energy between the chains in specimen 2\# at different strains and temperatures is shown in Figure 5. At $300 \mathrm{~K}$, the interaction energy between the chains sharply decreases from $10 \%$ strain to $20 \%$ strain, which indicates the occurrence of plastic deformation. However, no remarkable change in energy is observed at $500 \mathrm{~K}$ during the tensile process, which is consistent with the stress-strain curve. In addition, MD simulations can give insight into the deformation of the molecular structure during the loading process. Figure 6 shows a comparison of internal images (whole and zoomed in) of the simulated system under elastic and plastic deformations. It can be seen that the molecular structure in Figure 6(a) shows nearly homogeneous tension in the elastic stage. After plastic deformation, however, various failure modes, such as voids and disengaged junctions in the chains, could be found in the molecular structure in Figure 6(b).

The mechanical properties and shape memory effect of SMPs is strongly influenced by temperature. Figures 7 and 8 present snapshots of the molecular structures of the material under uniaxial stretches in the glassy and rubbery states, respectively. Although the initial configurations are similar, the deformation images of the material in the glassy and rubbery states are remarkably different. As shown in Figure 7 , the chains of the system are nearly homogeneously deformed in the glassy state. However, in the rubbery state, as shown in Figure 8, many voids are found in the structure

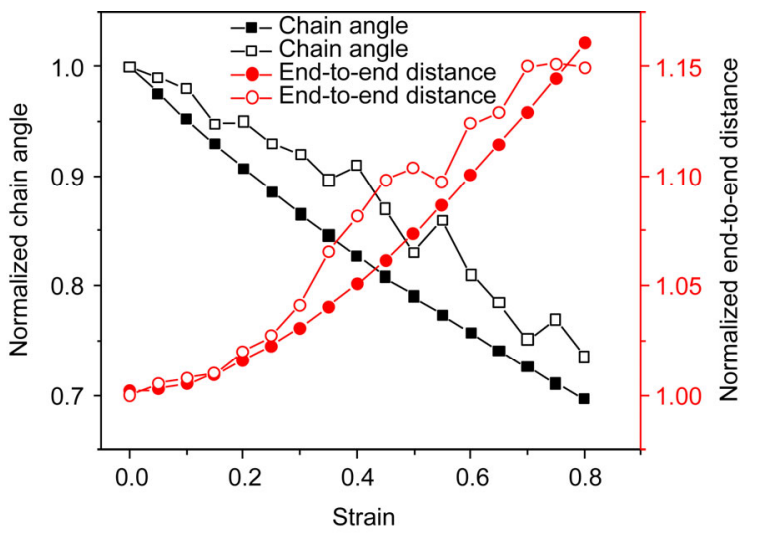

Figure 4 (Color online) End-to-end distance and chain orientation from MD simulations and theoretical predictions. 

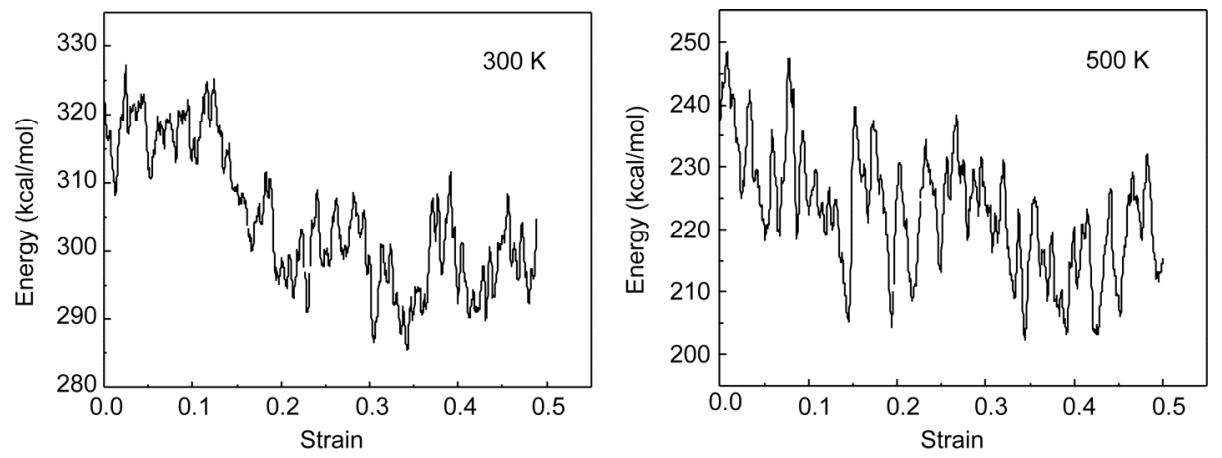

Figure 5 Interaction energy between chains at different temperatures (specimen 2\#).

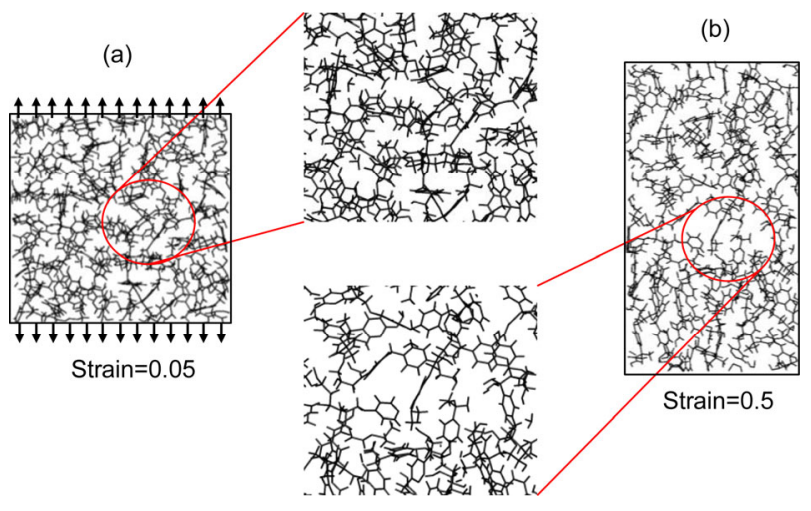

Figure 6 (Color online) Microstructures of the material (specimen 2\#) under elastic (a) and plastic deformations (b) at $300 \mathrm{~K}$.

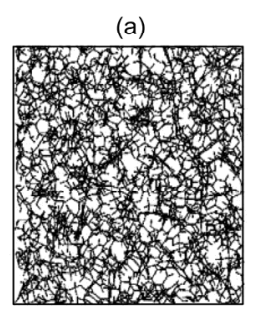

Strain $=0$

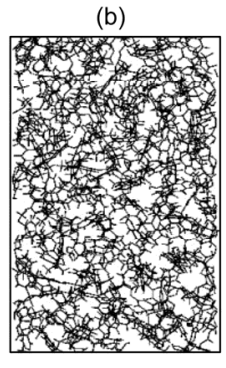

Strain $=0.2$ (c)

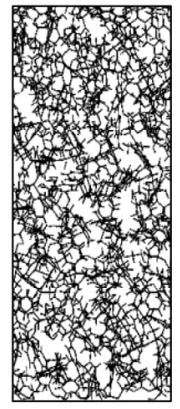

Strain $=0.5$
Figure 7 Snapshots of a representative epoxy SMP (specimen 2\#) under uniaxial stretches in the glassy state $(T=300 \mathrm{~K})$.

when the material is under plastic deformation. This might be due to different deformation mechanisms of SMP chains at high and low temperatures. The radius of gyration was used to characterize the structure of the molecules in the polymers. The radius of gyration of the three specimens in the glassy and rubbery states at 50\% strain is shown in Figure 9. These results show that the mean square radius of gyration is smaller in the rubbery state than that in the glassy state, which indicates that the chains are more flexible in the rubbery state (Figure 8), and not all of the chains are loaded, which leads to heterogeneous deformation and low yield stress (Figure 3). On the contrary, the lager mean square radius of gyration in the glassy state indicates that the chains are more entangled (Figure 7), and almost every chain is loaded during the deformation, which leads to homogeneous deformation and high yield stress (Figure 3 ).

\subsection{Shape frozen and recovery response}

To investigate the shape memory effect, we employed a thermomechanical cycle for the MD samples. As shown in

(c) (b)

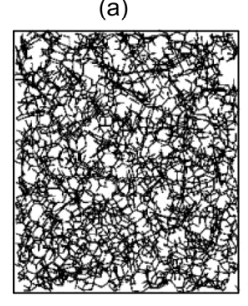

Strain $=0$

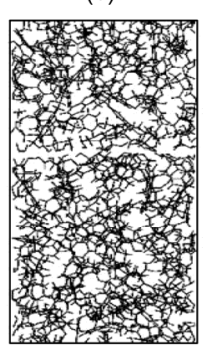

Strain $=0.2$

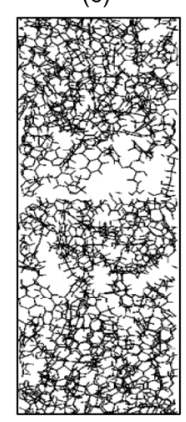

Strain $=0.5$
Figure 8 Snapshots of a representative epoxy SMP (specimen 2\#) under uniaxial stretches in the rubbery state $(T=500 \mathrm{~K})$.

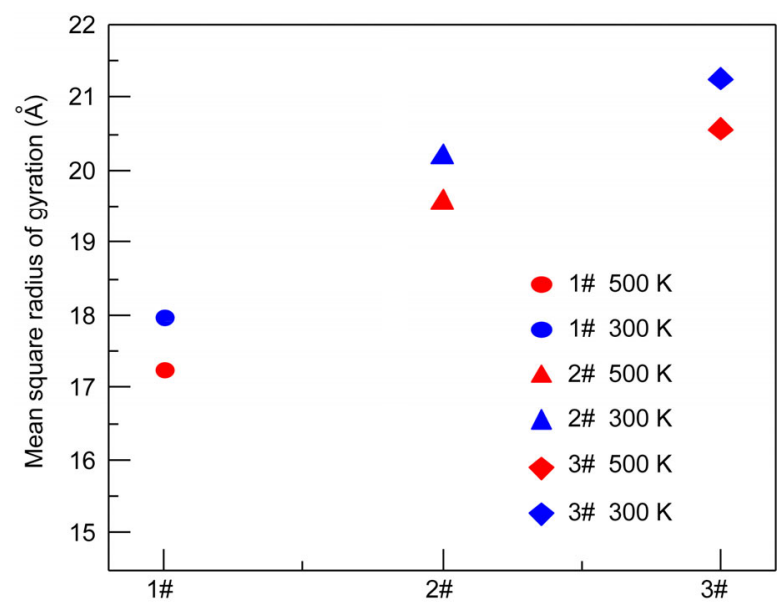

Figure 9 (Color online) Mean square radius of gyration of the three specimens in the glassy $(300 \mathrm{~K})$ and rubbery states $(500 \mathrm{~K})$. 
Figure 10, the thermomechanical cycle includes four steps: a uniaxial stretch above $T_{\mathrm{g}}$, a cooling step at a constant uniaxial stretch, a stress release step below $T_{\mathrm{g}}$, and finally a heating step. In the first step, the relaxed specimens $1 \#$ to $3 \#$ were uniaxially stretched $20 \%$ above $T_{\mathrm{g}}(400 \mathrm{~K}$ for specimens $1 \#$ and $2 \#, 450 \mathrm{~K}$ for specimen $3 \#)$, and then the stretched specimens were subjected to a 2 ns relaxation under an NVT ensemble to reach equilibrium conditions. We assumed that all the specimens have isotropic incompressible mechanical behavior [31]. Subsequently, in the shape memory cooling step, we employed an NVT ensemble to keep the volume of the stretched system constant. To limit the volume constraint, we adjusted the transverse dimensions of the box by setting the volume of the box equal to the volume of the undeformed box at the same temperature. Thus, the specimens were cooled down to $300 \mathrm{~K}$ under an NVT ensemble with a cooling rate of $50 \mathrm{~K} / \mathrm{ns}$. Because the internal pressure of the specimens cannot be released through long relaxation simulations at low temperatures, we employed an NPT ensemble with the Parrinello-Rahman barostat to release internal stress after the cooling step, setting the temperature to $300 \mathrm{~K}$ and the pressure to $0 \mathrm{~Pa}$. Subsequently, we heated the system to $T_{\mathrm{g}}$ using a long NPT simulation to investigate the recovery effect of SMPs at $T_{\mathrm{g}}$. Finally, the temperature was raised to the original value with an NPT simulation, and the strain recovery at three typical temperatures was obtained.

The stretch recovery during shape storage (low temperature) and shape recovery (high temperature) processes is shown in Figure 11. With increasing simulation time, no detectable recovery of the specimens could be observed at $300 \mathrm{~K}$ (black dots, Figure 11). That means that the structure is frozen and the materials exhibit ideal shape storage effects at room temperature. However, when the temperature was increased above $T_{\mathrm{g}}$, the specimens showed slow and steady recovery with increasing simulation time (red dots, Figure 11).

The same shape storage and recovery responses were also observed for all specimens. It is known that the time for shape recovery is highly dependent on $T_{\mathrm{g}}$ [44]: polymers with higher $T_{\mathrm{g}}$ values reform slower than those with lower $T_{\mathrm{g}}$ values. This trend is observed in Figure 11, with the fastest recovery for specimen $1 \#$ and the slowest for specimen $3 \#$, even though the recovery temperature is higher. This occurs because at a given recovery temperature, chain motion within polymers with a lower $T_{\mathrm{g}}$ is less restricted.

\section{Conclusions}

In summary, MD simulations were used to investigate the

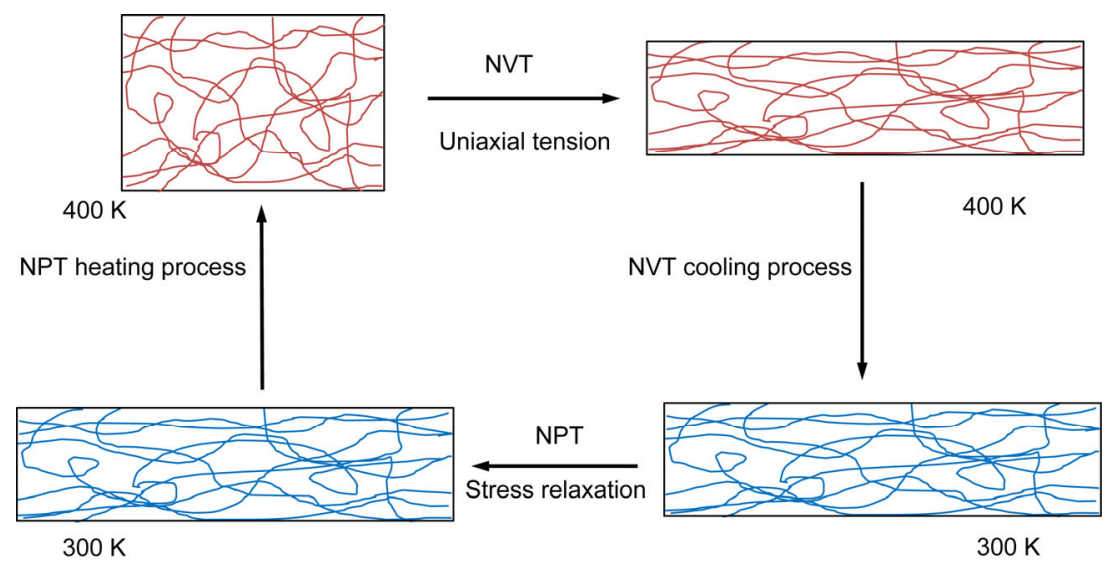

Figure 10 (Color online) Schematic thermomechanical loading process and the structure evolution for specimens.
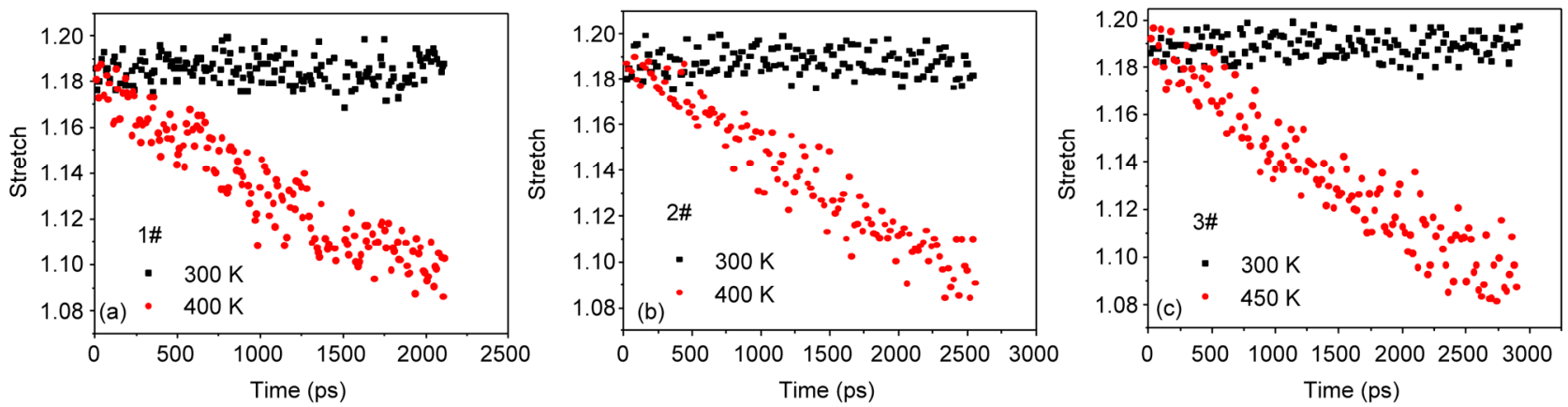

Figure 11 (Color online) Evolution of stretch recovery for specimens 1\# (a), 2\# (b), and 3\# (c) at different temperatures. 
thermomechanical properties and shape memory effect for three epoxy SMPs with varying contents of curing agent. The following results have been obtained:

(1) MD simulations revealed that the chains are homogeneously stretched along the loading direction during elastic deformation. However, during plastic deformation, various failure modes are found in the molecular structure, such as voids and disengaged junctions in the chains.

(2) The deformation of epoxy SMP resins in the rubbery state is inhomogeneous because the chains are flexible and their bonding is weak. In the glassy state, the chains in the system are homogeneously deformed along the loading direction because the chains become confined and their bonding is stronger.

(3) MD simulations of three types of SMPs revealed ideal shape storage at low temperature and shape recovery at high temperature. Furthermore, the recovery time is strongly influenced by the ratio of E-51 to DDM with more time needed for shape recovery as the ratio of curing agent increases.

This work was supported by the National Natural Science Foundation of China (Grant Nos. 11272044 and 11023001).

1 A. Lendlein, and S. Kelch, Angew. Chem. Int. Edit. 41, 1 (2002).

2 H. Jiang, S. Kelch, and A. Lendlein, Adv. Mater. 18, 1471 (2006).

3 H. Koerner, G. Price, N. A. Pearce, M. Alexander, and R. A. Vaia, Nat. Mater. 3, 115 (2004).

4 A. Lendlein, H. Jiang, O. Jünger, and R. Langer, Nature 434, 879 (2005).

5 A. M. Schmidt, Macromol. Rapid Comm. 27, 1168 (2006).

6 Z. He, N. Satarkar, T. Xie, and Y. T. Cheng, J. Z. Hilt, Adv. Mater. 23, 3192 (2011).

7 W. M. Huang, B. Yang, L. An, C. Li, and Y. S. Chan, Appl. Phys. Lett. 86, 114105 (2005).

8 Y. C. Jung, H. H. So, and J. W. Cho, J. Macromol. Sci. B 45, 453 (2006).

9 B. K. Kim, S. Y. Lee, and M. Xu, Polymer 37, 5781 (1996).

10 D. Pérez-Foullerat, S. Hild, A. Mücke, and B. Rieger, Macromol. Chem. Phys. 205, 374 (2004).

11 A. L. Browne, and N. L. Johnson, Shape Memory Polymer Seat Assemblies, US patent, 7309104 (2007-12-18).

12 S. Chen, J. Hu, Y. Liu, H. Liem, Y. Zhu, and Q. Meng, Polym. Int. 56, 1128 (2007).

13 K. Gall, M. L. Dunn, Y. Liu, D. Finch, M. Lake, and N. A. Munshi, Acta Mater. 50, 5115 (2002).

14 J. W. Cho, J. W. Kim, Y. C. Jung, and N. S. Goo, Macromol. Rapid
Comm. 26, 412 (2005).

15 W. M. Huang, J. Int. Mat. Syst. Str. 17, 753 (2006).

16 M. A. D. Prima, M. Lesniewski, K. Gall, D. L. McDowell, T. Sanderson, and D. Campbell, Smart Mater. Struct. 16, 2330 (2007).

17 Z. F. Li, and Z. D. Wang, J. Int. Mat. Syst. Str. 22, 1605 (2011).

18 Z. D. Wang, and Z. F. Li, Arch. Appl. Mech. 81, 1667 (2011).

19 I. Rao, and K. Rajagopal, Int. J. Solids Struct. 38, 1149 (2001).

20 H. Tobushi, K. Okumura, S. Hayashi, and N. Ito, Mech. Mater. 33, 545 (2001).

21 Y. Chen, and D. Lagoudas, J. Mech. Phys. Solids 56, 1752 (2008).

22 Z. Wang, Z. Li, L. Wang, and Z. Xiong, J. Appl. Polym. Sci. 118, 1406 (2010).

23 H. Lu, Y. Liu, J. Gou, J. Leng, and S. Du, Int. J. Smart Nano Mater. 1, 2 (2010).

24 Y. Liu, K. Gall, M. L. Dunn, A. R. Greenberg, and J. Diani, Int. J. Plasticity 22, 279 (2006).

25 I. Bellin, S. Kelch, R. Langer, and A. Lendlein, Proc. Natl. Am. Sci. 103, 18043 (2006).

26 T. Xie, X. Xiao, and Y. T. Cheng, Macromol. Rapid Commun. 30, 1823 (2009).

27 T. Pretsch, Smart Mater. Struct. 19, 427 (2010).

28 Z. Wang, W. Song, L. Ke, and Y. Wang, Mater. Lett. 89, 216 (2012).

29 S. S. Jang, W. A. Goddard, and M. Y. S. Kalani, J. Phys. Chem. B 111, 1729 (2007).

30 C. Li, and A. Strachan, Polymer 52, 2920 (2011).

31 J. Diani, and K. Gall, Smart Mater Struct. 16, 1575 (2007).

32 E. Ghobadi, M. Heuchel, K. Kratz, and A. Lendlein, Macromol. Chem. Phys. 214, 1273 (2013).

33 M. Tupper, N. Munshi, F. Beavers, K. Gall, M. Mikuls, and T. Meink, IEEE Proc. 5, 2541 (2001).

34 D. Campbell, M. S. Lake, M. R. Scherbarth, E. Nelson, and R. W. Six, in 46th AIAA/ASME/ASCE/AHS/ASC Structures, Structural Dynamics and Materials Conference, Austin, USA, 2005, AIAA 2005-2362.

35 C. S. Hazelton, K. R. Gall, E. R. Abrahamson, R. J. Denis, and M. S. Lake, in 44th AIAA/ASME/ASCE/AHS/ASC Structures, Structural Dynamics and Materials Conference, Norfolk, USA, 2003, AIAA 2003-1977.

36 W. Francis, M. S. Lake, K. Mallick, and G. E. Freebury, A. Maji, in 44th AIAA/ASME/ASCE/AHS/ASC Structures, Structural Dynamics and Materials Conference, Norfolk, USA, 2003, AIAA 2003-1496.

37 W. B. Song, L. L. Wang, and Z. D. Wang, Mater. Sci. Eng. A-Struct. 529, 29 (2011).

38 Accelrys Materials Studio, Version 5.0 (Accelrys Software Inc., San Diego (CA), 2009).

39 S. Plimpton, J. Comput. Phys. 117, 1 (1995).

40 H. Sun, Macromolecules 28, 701 (1995).

41 H. B. Fan, and M. M. F. Yuen, Polymer 48, 2174 (2007).

42 P. Wu, and E. Van der Giessen, J. Mech. Phys. Solids. 41, 427 (1993).

43 P. Wu, and E. Van der Giessen, Mech. Res. Commun. 19, 427 (1992).

44 C. M. Yakacki, R. Shandas, C. Lanning, B. Rech, A. Eckstein, and K. Gall, Biomaterials 28, 2255 (2007). 